\title{
Generalized Scaling of Urban Heat Island Effect and Its Applications for Energy Consumption and Renewable Energy
}

\author{
T.-W. Lee, ${ }^{1}$ Heung S. Choi, ${ }^{2}$ and Jinoh Lee ${ }^{1}$ \\ ${ }^{1}$ Department of Mechanical and Aerospace Engineering, Arizona State University, Tempe, AZ 85287-6106, USA \\ ${ }^{2}$ Department of Mechanical and Design Engineering, Hongik University, Sejong, Republic of Korea \\ Correspondence should be addressed to T.-W. Lee; attwl@asu.edu
}

Received 14 March 2014; Accepted 9 June 2014; Published 18 August 2014

Academic Editor: Huei-Ping Huang

Copyright (c) 2014 T.-W. Lee et al. This is an open access article distributed under the Creative Commons Attribution License, which permits unrestricted use, distribution, and reproduction in any medium, provided the original work is properly cited.

\begin{abstract}
In previous work from this laboratory, it has been found that the urban heat island intensity (UHI) can be scaled with the urban length scale and the wind speed, through the time-dependent energy balance. The heating of the urban surfaces during the daytime sets the initial temperature, and this overheating is dissipated during the night-time through mean convection motion over the urban surface. This may appear to be in contrast to the classical work by Oke (1973). However, in this work, we show that if the population density is used in converting the population data into urbanized area, then a good agreement with the current theory is found. An additional parameter is the "urban flow parameter," which depends on the urban building characteristics and affects the horizontal convection of heat due to wind. This scaling can be used to estimate the UHI intensity in any cities and therefore predict the required energy consumption during summer months. In addition, all urbanized surfaces are expected to exhibit this scaling, so that increase in the surface temperature in large energy-consumption or energy-producing facilities (e.g., solar electric or thermal power plants) can be estimated.
\end{abstract}

\section{Introduction}

There have been many studies on the causes and impact of the heat island effect (e.g., [1-9]). A wide range of causes have been attributed, including the land surface energy balance (or imbalance) due to urbanized land surface and built structures, anthropogenic heat release, and different atmospheric constituents over the city $[10,11]$. While the significance of the urban heat island effect is universally recognized $[12,13]$, the approaches to its understanding and mitigation are quite varied, ranging from simple "slab" approach [14] to sophisticated models that resolve urban canopy physics $[15,16]$. One of the important aspects is quantifying the magnitude of the urban heat island effect in terms of relevant variables, which again vary widely depending on the authors. A key step in this quantification is "scaling," that is, developing a functional relationship between the urban heat island effect and causal variables.

In previous work from this laboratory, we have shown that scaling of the urban heat island effect based on timedependent energy balance works quite well in understanding and predicting the UHI dependence on the urban length scale and wind speeds [17]. This approach is in contrast to the scaling used by many authors [18-24], where a steady-state energy balance is used along with horizontal and vertical heat flux terms. It is clear that the urban island heating (as well as cooling) is not a steady-state effect, nor is the UHI intensity determined at some fixed time, as shown in Figure 1 where a typical time evolution of the UHI intensity is plotted. In this work, we show that if the population density is used in converting the population data into urbanized area in Oke's work [9], then a good agreement with the current theory is found. An additional parameter is the "urban flow parameter," which depends on the urban building characteristics and affects the horizontal convection of heat due to wind. This scaling can be used to estimate the UHI intensity in any cities and therefore predict the required energy consumption during summer months. In addition, all urbanized surfaces are expected to exhibit this scaling, so that increase in the surface temperature in large energy-consumption or energyproducing facilities (e.g., solar electric or thermal power plants) can be estimated. About one-third of the electric 


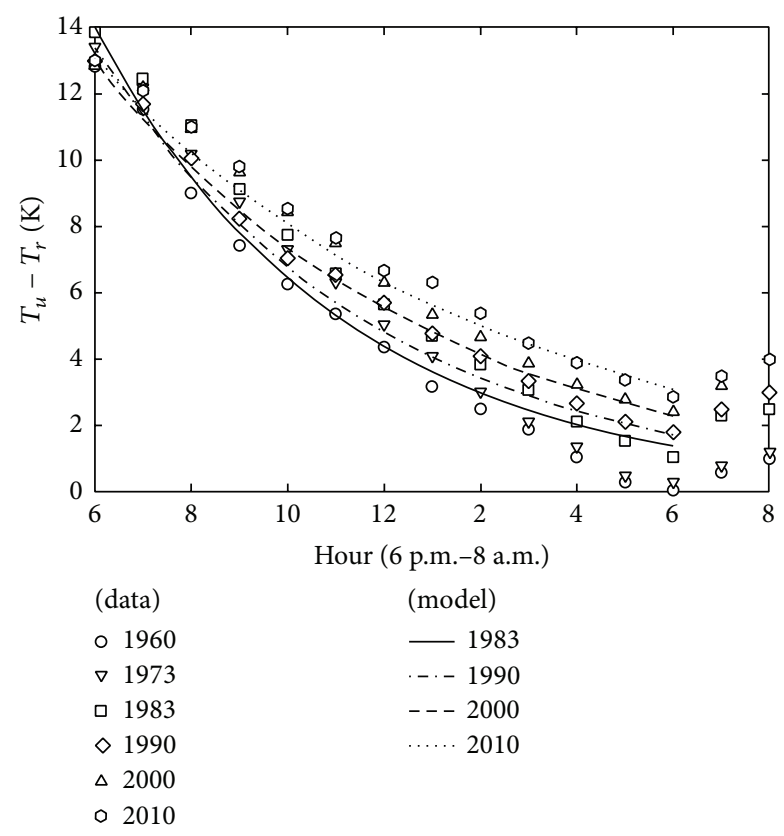

FIgure 1: Temperature decay as a function of time, in Phoenix, AZ, for years 1960 to 2010, using (3).

power goes to satisfy the residential consumption in United States, and thus understanding of the electricity demand due to UHI increase is a key component in planning for renewable energy production.

\section{Methods of Analyses}

A brief summary of the scaling is given below. The starting point is the fundamental energy balance equation [17]:

$$
\rho c_{p} \frac{\partial T_{u}}{\partial t}+\rho c_{p} U \frac{\partial T_{u}}{\partial x}=\dot{q}^{\prime \prime}
$$

where $q^{\prime \prime}$ is the divergence of the eddy-diffusive heat flux. The air density and specific heat are $\rho$ and $c_{p}$, respectively, while $U$ is the convection speed, often approximated as the mean wind velocity over the urban area. The spatial gradient in the temperature can be approximated and moved to the right-hand side. In our previous work [17], we considered the time evolution of the urban temperature during night hours, after the heat flux from the surface has led to some "equilibrium" temperature above the urban surface. This argument considers the heat flux term to set some initial urban temperature, prior to subsequent cooling, and thus heat flux term in (1) has been removed and absorbed into the initial temperature difference $\left(T_{u}-T_{r}\right)_{o}$. For now, we can retain this term, as it can have additive effect on the urban air temperature during the night hours. The temperatures, $T_{u}$ and $T_{r}$, represent temperatures in the urban and rural locations, respectively, and $\left(T_{u}-T_{r}\right)$ has been used as the UHI intensity:

$$
\frac{\partial T_{u}}{\partial t} \approx-U \frac{T_{u}-T_{r}}{L}+\frac{\dot{q}^{\prime \prime}}{\rho c_{p}} .
$$

If we integrate while retaining only the first term on the right-hand side, we obtain the temporal evolution of the UHI intensity. This assumes that the convection effect is much stronger than the heat flux term:

$$
\Delta T(t)=T_{u}-T_{r}=\left(T_{u}-T_{r}\right)_{o} \exp \left(-K \frac{U}{L} t\right)
$$

where $K=1$ in the limiting case of $q^{\prime \prime}=0$ and $K<1$ in general, if the diffusive heat flux retarding the night cooling process is taken into account. This exponential decay in the temperature difference is closely reflected by typical data for time evolution at night hours in Figure 1.

On the other hand, if we consider the other extreme case of zero wind speed and retain only the heat flux term, then we obtain

$$
T_{u}-T_{r}=\left(T_{u}-T_{r}\right)_{o}+\frac{\dot{q}^{\prime \prime}}{\rho c_{p}} t
$$

From (3), we can see that the wind speed, $U$, has the obvious effect of enhancing the cooling of the heated air over the urban surface. The larger the urban length scale is, $L$, the longer it would take to cool $T_{u}$. The heat flux term, as shown in (4), adds to the urban temperature until the heat is completely dissipated. The urban-rural breeze usually results in an enhanced convection motion due to mean wind in urban areas, which dominates the heat transfer process over the heat flux term due to eddy diffusion. For compactness, we retain the solution form of (4) and use a correction factor, $K$, to reflect the facts that (1) the temperature decay will be delayed by the heat flux term and (2) different urban surface morphologies, aerodynamic properties, and atmospheric stability may affect the effectiveness of wind speed in cooling of the urban temperature. Thus, a large heat flux term and structural impedance to convection cooling will both result in smaller value of $K$. We can observe from (3) then that the cooling should undergo an exponential decay as a function of time (Figure 1), and depending on the above factors the final minimum temperature achieved will be the so-called night-time minimum temperature typically reported in meteorological data sets. In addition, the wind speed also should lead to an exponential decrease in UHI. A large set of data has been used to validate this model, and an example is shown in Figure 2 where the wind speed effect for various urban areas is shown to follow the exponential decrease according to (3).

In this work, we have used the data reported by Oke [9], along with the population and urban surface area data dated at the same time period (1968-1978), in order to offer further proof that the correct scaling for the UHI intensity should be based on time-dependent energy balance and that there is exponential dependence as a function of time, wind speed, and the urban length scale. The following Canadian cities are considered as in Oke [9]: Montreal, St. Hyacinthe, St. Hubert, Chambly, Marieville, St. Basile-Le Grand, St. Cesaire, St. Pie, Ste. Angele-de Monnoir, and Ste. Madeline. 


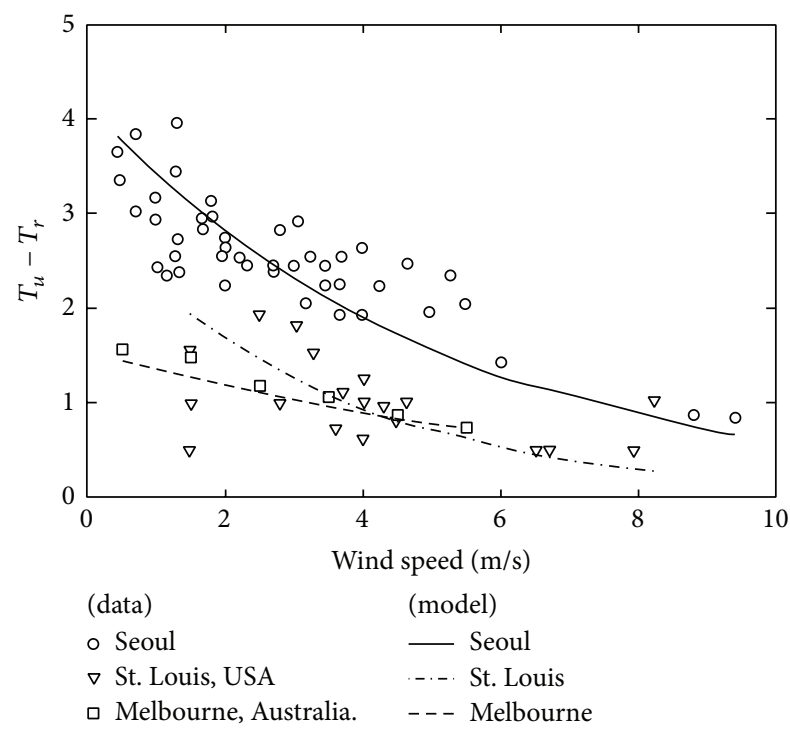

FIGURE 2: The effect of the wind speed on the UHI intensity in Seoul, Korea, St. Louis, USA, and Melbourne, Australia. The theoretical lines are obtained using (3). $\left(T_{u}-T_{r}\right)$ is the UHI intensity defined as the maximum difference in the temperatures in the urban and rural locations.

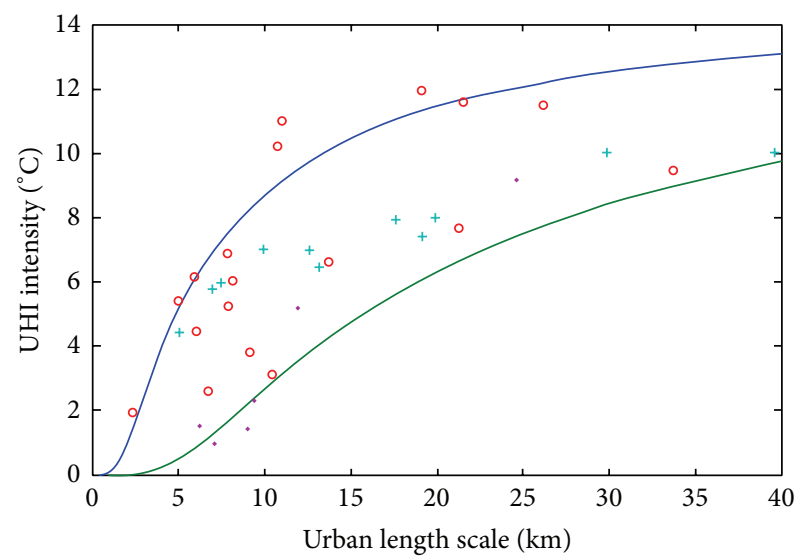

FIGURE 3: UHI intensity as a function of the urban length scale. Symbols represent data as reported by Oke [9]. The upper curve is (3) with $K=1.0$, while the lower is for $K=0.25$.

\section{Results}

Figure 3 shows the UHI intensity as a function of the urban length scale, for all of the cities reported in Oke [9]. In Oke's work, the UHI intensity was correlated and plotted as a function of the population ("city size"), but no sound physical basis was given. Other authors have attempted a similar correlation, but different slopes were required for North American, European, Japanese, and Korean cities. Figure 3 shows that the UHI intensity for Canadian cities can be plotted using the current scaling, within the upper $(K=1)$ and lower $(K=0.25)$ bounds, using the urban area converted to the urban length scale of the identical time periods. As noted above and in our previous work, the parameter $K$

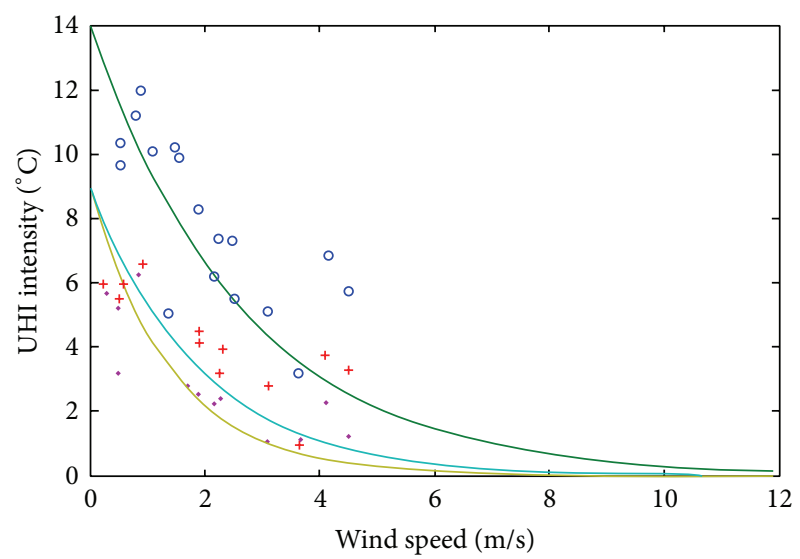

FIGURE 4: The effect of the wind speed on the UHI intensity in Chambly, Marieville, St. Basile-Le Grand, St. Cesaire, St. Pie, Ste. Angele-de Monnoir, and Ste. Madeline. Symbols represent data as reported by Oke [9], while the theoretical lines are obtained from (3).

depends on the urban morphological characteristics. Thus, there is a direct effect of increase in the urban length scale on the UHI intensity. In our previous work [17], we have investigated the growth of two cities, Phoenix and Tucson, based on the satellite image analyses, and corresponding increase in the urban length scale. Of course, the growth of urban areas depends on many economical and sociological parameters, as the expected growth of Phoenix, for example, has been halted in 2008 due to housing meltdown.

Thus far, in this and our previous work [17], we have looked at cities around the world with widely disparate population densities, urban surface morphology, and meteorological conditions. Although the general UHI characteristics are captured by (3), exact functional relationships tend to vary as shown in Figure 3 even for cities within Canada. Thus, incorporating and categorizing the effects of urban surface morphology and meteorological conditions into the parameter $K$ in (3) remain as the next task in this formation for the UHI scaling.

Figures 4, 5, and 6 show the effects of the wind speed on the UHI intensity for the same Canadian cities as in Figure 3. It can be observed that functional relationship between the UHI intensity and the wind speed is accurately captured using (3). Hunt et al. [25] have also looked at the scaling of UHI effects for large urban areas and attempted to link the worsening of UHI effects on global warming. As shown in (3), the initial temperature difference between urban and rural areas does determine the final UHI intensity. However, the more direct factors are the urban length scale and the wind speed, since the global warming would elevate the temperatures in urban and rural areas uniformly.

\section{Conclusions}

Effects of the urban length scale and the wind speed on the urban heat island effect can be quantified through timedependent energy balance. The heating of the urban surfaces 


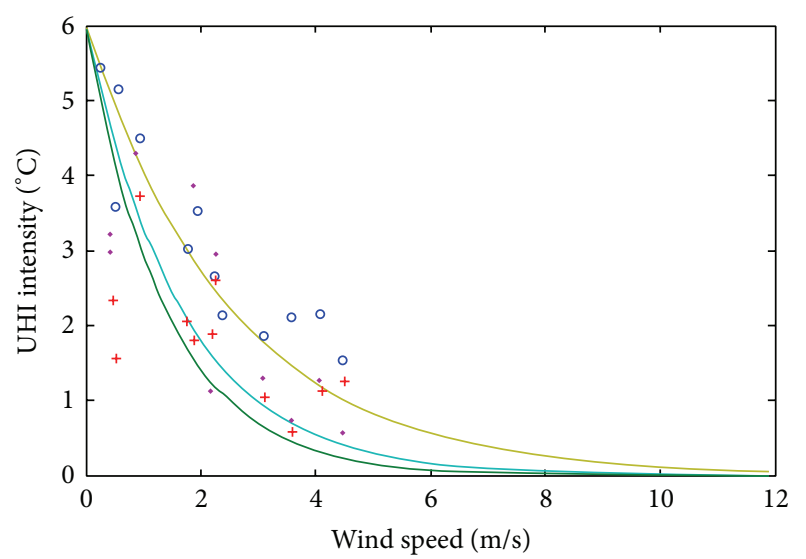

Figure 5: The effect of the wind speed on the UHI intensity in Chambly, Marieville, and St. Basile-Le Grand.

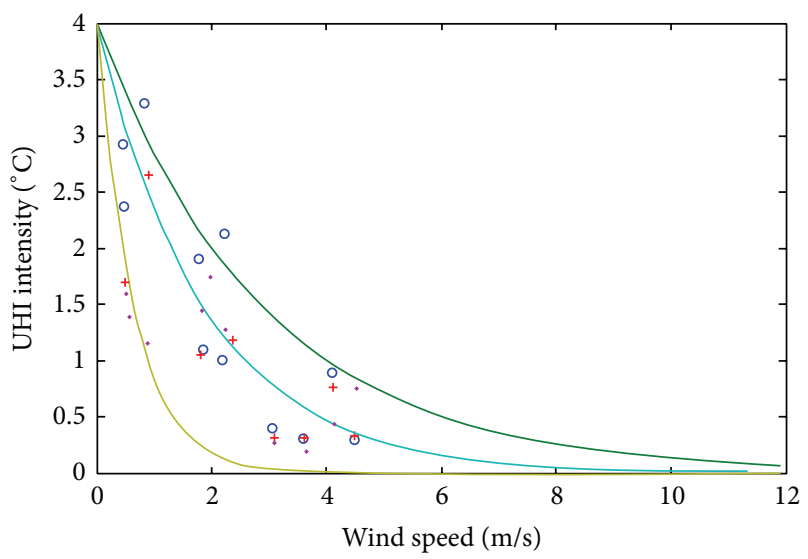

FIGURE 6: The effects of wind speed on UHI intensity for St. Cesaire, St. Pie, and Ste. Angele-de Monnoir.

during the daytime sets the initial temperature, and this overheating is cooled during the nighttime through mean convection motion over the urban surface, resulting in an exponential decay in the temperature. This overheating is cooled during the nighttime, through mean convection motion over the urban surface, resulting in an exponential decay in the temperature. The solution to the time-dependent energy balance equation reproduces this temporal decay with good accuracy, with the main factors being the length scale of the urban area and the wind speed. The minimum temperature reached at the end of night-time cooling period then corresponds to the UHI intensity, which increases with increasing urban length scale and decreasing wind speed. The wind speed effect is also accurately retraced using this method; however, different correction factors are required for different cities, indicating the effects of the urban surface heat content, structural morphology, and density. Thus, using a small number of readily available data for the urban length scale and the wind speed, the UHI intensity can be described with possible projections for future trends. This approach can be used in planning of energy resources, as well as any large areas of concrete surfaces needed for renewable or other power generation. About one-third of the electric power goes to satisfy the residential consumption in United States, and thus understanding of the electricity demand due to UHI increase is a key component in planning for renewable energy production.

\section{Conflict of Interests}

The authors declare that there is no conflict of interests regarding the publication of this paper.

\section{References}

[1] L. A. Baker, A. J. Brazel, N. Selover et al., "Urbanization and warming of Phoenix (Arizona, USA): impacts, feedbacks and mitigation," Urban Ecosystems, vol. 6, pp. 183-203, 2002.

[2] A. Brazel, N. Selover, R. Vose, and G. Heisler, "The tale of two climates: baltimore and phoenix urban LTER sites," Climate Research, vol. 15, no. 2, pp. 123-135, 2000.

[3] J. R. Christy, W. B. Norris, K. Redmond, and K. P. Gallo, "Methodology and results of calculating central California surface temperature trends: evidence of human-induced climate change?" Journal of Climate, vol. 19, no. 4, pp. 548-563, 2006.

[4] A. T. J. de Laat and A. N. Maurellis, "Evidence for influence of anthropogenic surface processes on lower troposheric and surface temperature trends," International Journal of Climatology, vol. 26, no. 7, pp. 897-913, 2006.

[5] E. Kalnay and M. Cai, "Impact of urbanization and land-use change on climate," Nature, vol. 423, no. 6939, pp. 528-531, 2003.

[6] D. Pearlmutter and P. Berliner, "Street canyon geomtry and microclimate: designing for urban comfort under arid conditions," in Environmentally Friendly Cities, E. Maldonado and S. Yannas, Eds., Proceedings of PLEAS '98, pp. 165-166, Lisbon, Portugal, 1998.

[7] Y. Rydin, "Environmental dimensions of residential development and the implications for local planning practice," Journal of Environmental Planning \& Management, vol. 35, no. 1, pp. 4361, 1992.

[8] B. Stone Jr. and M. O. Rodgers, "Urban form and thermal efficiency how the design of cities influences the Urban heat island effect," Journal of the American Planning Association, vol. 67, no. 2, pp. 186-198, 2001.

[9] T. R. Oke, "City size and the urban heat island," Atmospheric Environment, vol. 7, no. 8, pp. 769-779, 1973.

[10] H. E. Landsberg, Atmospheric Changes in a Growing Community, Technical Note-Institute for Fluid Dynamics and Applied Mathematics, BN 823, Institute for Fluid Dynamics and Applied Mathematics, University of Maryland, College Park, Md, USA, 1975.

[11] D. J. Sailor, "A review of methods for estimating anthropogenic heat and moisture emissions in the urban environment," International Journal of Climatology, vol. 31, no. 2, pp. 189-199, 2011.

[12] A. J. Arnfield, "Two decades of urban climate research: a review of turbulence, exchanges of energy and water, and the urban heat island," International Journal of Climatology, vol. 23, no. 1, pp. 1-26, 2003.

[13] W. T. L. Chow, D. Brennan, and A. J. Brazel, "Urban heat island research in phoenix, Arizona: Theoretical contributions and policy applications," Bulletin of the American Meteorological Society, vol. 93, no. 4, pp. 517-530, 2012. 
[14] C. S. B. Grimmond, H. A. Cleugh, and T. R. Oke, "An objective urban heat storage model and its comparison with other schemes," Atmospheric Environment B: Urban Atmosphere, vol. 25, no. 3, pp. 311-326, 1991.

[15] F. Chen, H. Kusaka, R. Bornstein et al., "The integrated WRF/urban modelling system: development, evaluation, and applications to urban environmental problems," International Journal of Climatology, vol. 31, no. 2, pp. 273-288, 2011.

[16] Z.-H. Wang, E. Bou-Zeid, and J. A. Smith, "A spatially-analytical scheme for surface temperatures and conductive heat fluxes in urban canopy models," Boundary-Layer Meteorology, vol. 138, no. 2, pp. 171-193, 2011.

[17] T. W. Lee and A. Ho, "Scaling of the urban heat island effect based on the energy balance: nighttime minimum temperature increase vs. urban area length scale," Climate Research, vol. 42, no. 3, pp. 209-216, 2010.

[18] P. W. Summers, An urban ventilation model applied to Montreal [Ph.D. thesis], McGill University, 1964.

[19] I. Uno, S. Wakamatsu, H. Ueda, and A. Nakamura, "An observational study of the structure of the nocturnal urban boundary layer," Boundary-Layer Meteorology, vol. 45, no. 1-2, pp. 59-82, 1988.

[20] R. Richiardone and G. Brusasca, "Numerical experiments on urban heat island intensity," Quarterly Journal of the Royal Meteorological Society, vol. 115, no. 488, pp. 983-995, 1989.

[21] S. S. Raman and J. E. Cermak, "Physical modeling of flow and diffusion over an urban heat island," Advances in Geophysics, vol. 18, pp. 223-240, 1975.

[22] J. Lu, P. Arya, W. H. Snyder, and R. E. Lawson Jr., "A laboratory study of the urban heat island in a calm and stably stratified environment. Part I: temperature field," Journal of Applied Meteorology, vol. 36, no. 10, pp. 1377-1391, 1997.

[23] J. Lu, S. P. Arya, W. H. Snyder, and R. E. Lawson Jr., "A laboratory study of the urban heat island in a calm and stably stratified environment-part II-velocity field," Journal of Applied Meteorology, vol. 36, no. 10, pp. 1392-1402, 1997.

[24] A. Cenedese and P. Monti, "Interaction between an inland urban heat island and a sea-breeze flow: a laboratory study," Journal of Applied Meteorology and Climatology, vol. 42, pp. 1569-1583, 2003.

[25] J. C. Hunt, Y. V. Timoshkina, S. I. Bohnenstengel, and S. Belcher, "Implications of climate change for expanding cities worldwide," Proceedings of the ICE-Urban Design and Planning, vol. 166, no. 4, pp. 214-254, 2012. 

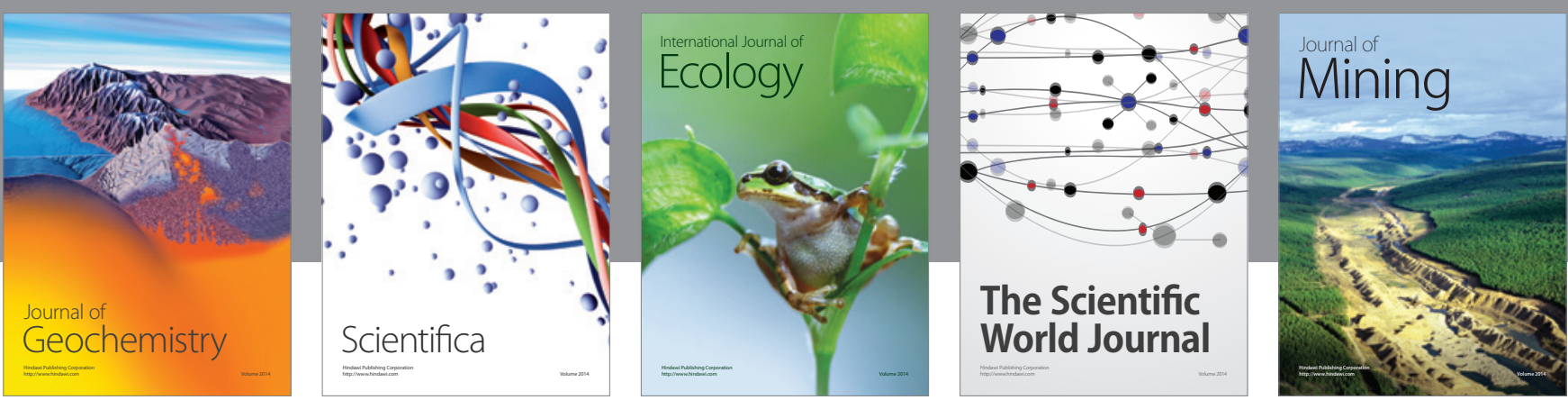

The Scientific World Journal
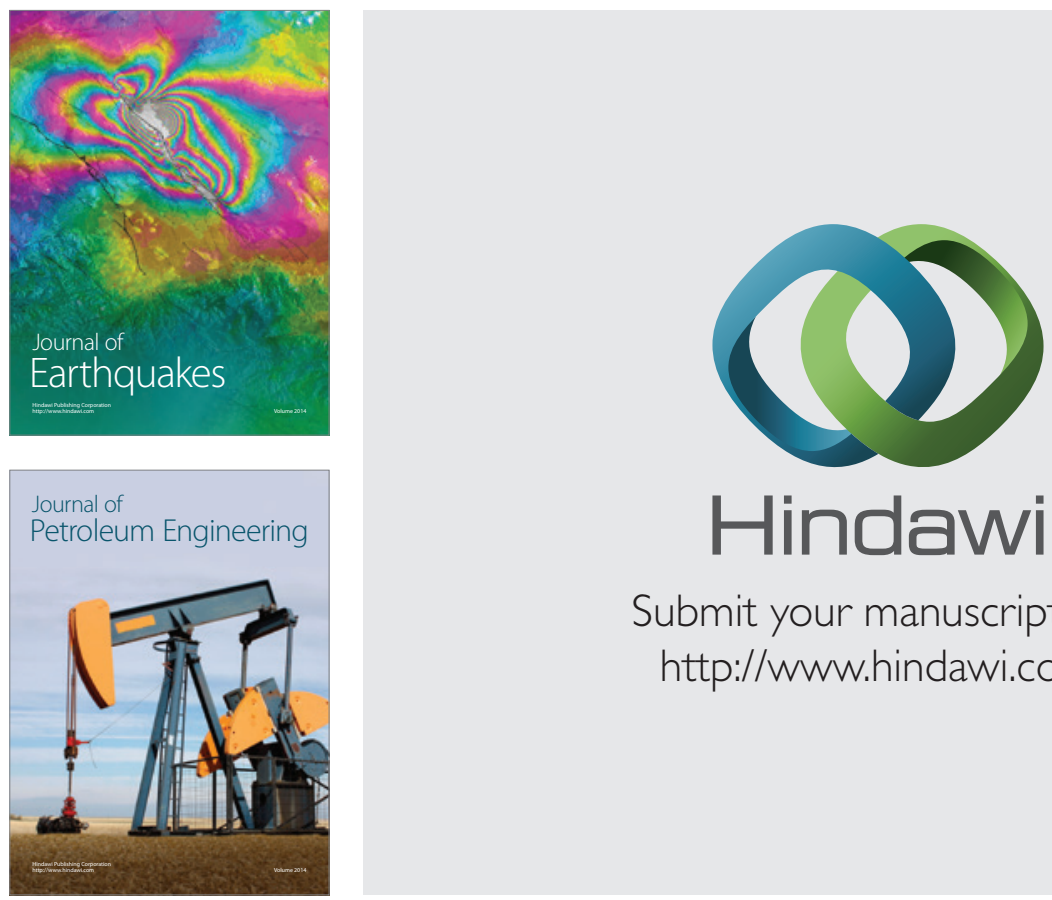

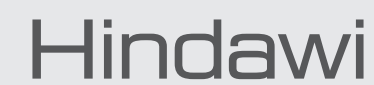

Submit your manuscripts at

http://www.hindawi.com
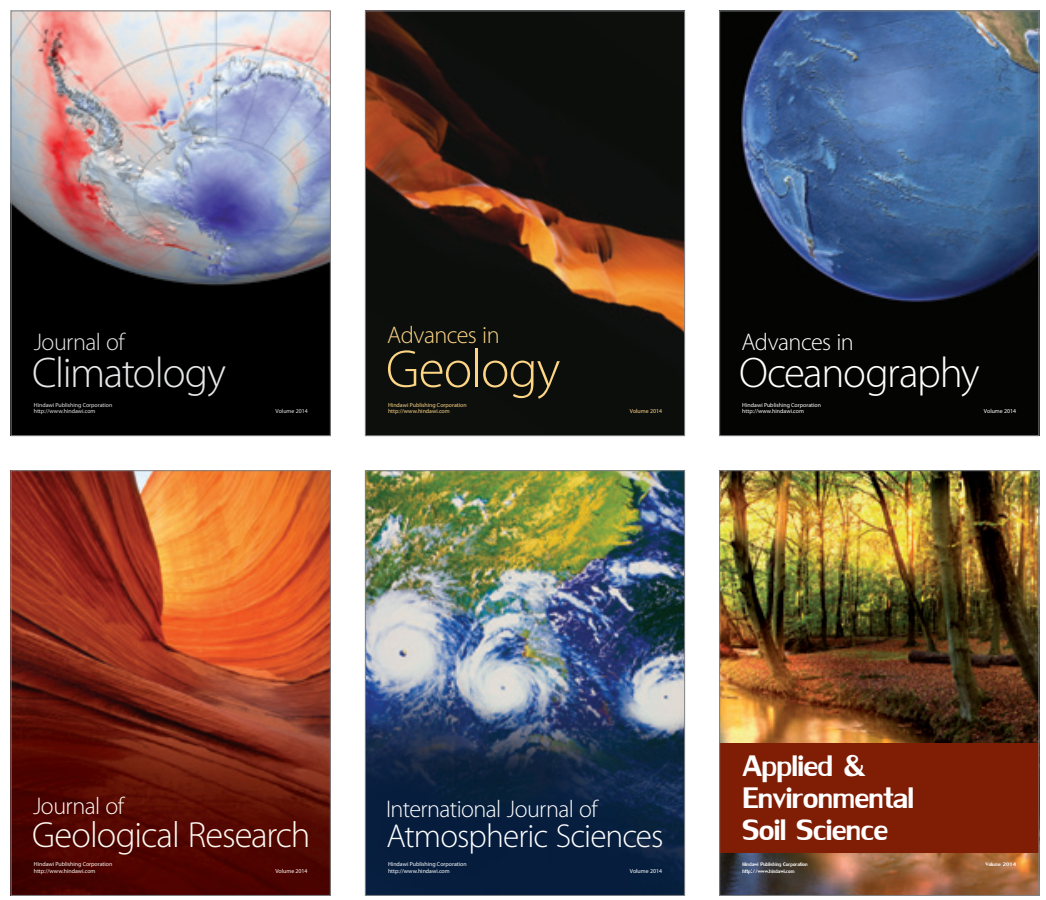
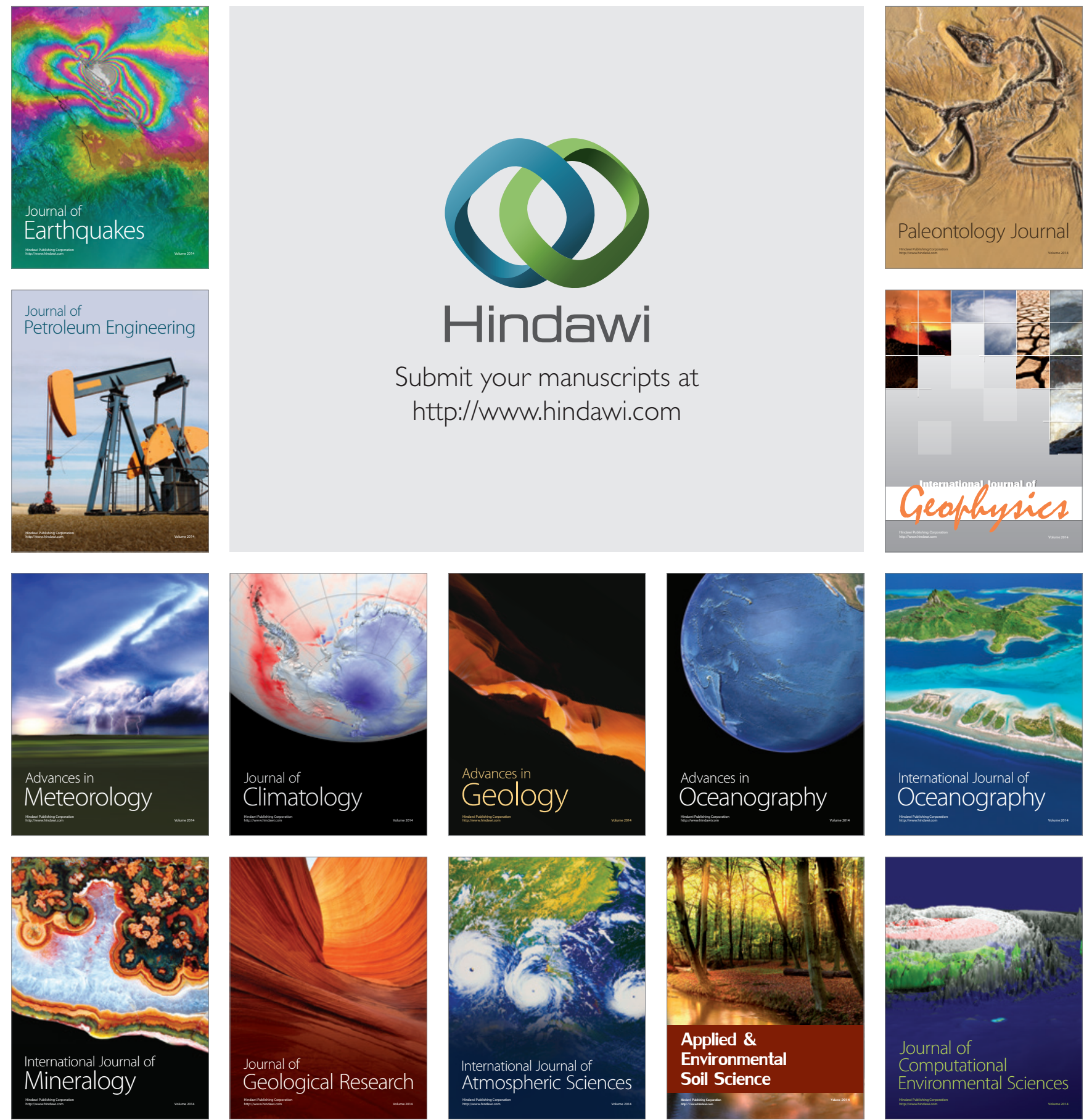